\title{
About diffusivity, radiative viscosity and particle transport
}

\author{
G. Alecian ${ }^{1}$ and G. Michaud ${ }^{2}$ \\ ${ }^{1}$ LUTH (Observatoire de Paris - CNRS), Observatoire de Meudon, 92195 Meudon Cedex, France \\ e-mail: georges.alecian@obspm.fr \\ 2 Département de Physique, Université de Montréal, Montréal, PQ, H3C 3J7, Canada \\ e-mail: michaudg@astro.umontreal.ca
}

Received 7 May 2004 / Accepted 10 October 2004

\begin{abstract}
It is shown that the process called radiative diffusivity by Morel \& Thévenin $(2002,390,611)$ does not exist as they describe it. Their description is based on a confusion between atomic diffusion and turbulent transport (often called turbulent diffusion in the context of particle transport in stars). We evaluate how ions are slowed down by photons and show that this may be neglected in atomic diffusion calculations. In their evolutionary model calculations, Morel \& Thévenin (2002) made the ad hoc hypothesis that turbulent diffusion was proportional to radiative viscosity.
\end{abstract}

Key words. diffusion - stars: general

\section{Introduction}

It has been suggested by Morel \& Thévenin (2002) that a process they called radiative diffusivity (see their Sect. 2) had been overlooked by Burgers (1969) and Paquette et al. (1986) in their calculations of atomic diffusion coefficients.

In their introduction (page 612 of their paper): "In this exploratory work, we focus on the role of the radiative diffusivity generated by the photon-ion collisions that is not presently taken into account in the microscopic diffusion coefficients [...] we present a simplified phenomenological model of the radiative diffusivity resulting from photon-ion collisions."

They base their argumentation on an analogy (page 613 of their paper). On the one hand, they note that the ion-proton atomic diffusion coefficient is proportional to molecular viscosity; on the other hand they indicate that radiative viscosity dominates molecular viscosity in many stars. They then assume that radiative viscosity should lead to what they called radiative diffusivity which they multiplied by an efficiency factor.

The authors give no other physical argument than the preceding analogy for the existence of radiative diffusivity. They then proceed to use it in evolutionary model calculations. They do not clearly state where the radiative diffusivity is introduced into their particle transport equations. They apparently introduce it as one would introduce turbulent transport (Schatzman 1969) since it has the effect of reducing surface abundance variations, which is the effect of turbulent transport. This is inconsistent with their claim quoted above that the process had been "overlooked in ...calculations of atomic diffusion coefficients".

In our opinion, the process the authors describe does not exist. Their description is based on a confusion between atomic diffusion and turbulent transport (often called turbulent diffusion). In the next section, we briefly show how atomic diffusion coefficients should be combined in multicomponent gases by taking the example of ions interacting with both protons and electrons. We then suggest intuitively how a photon gas may lead to a diffusion coefficient. We evaluate it roughly and compare it to atomic diffusion coefficients.

By examining how the authors apparently included radiative diffusivity in their calculations, we conclude in the last section that they made the ad hoc assumption that turbulent diffusion had the same parametrization as radiative viscosity.

\section{Atomic transport coefficients}

In multicomponent gases, each species interacts with all the others, so that calculating transport velocities requires including interactions with all species. In the case of a binary mixture with elastic binary collisions, the transport problem has been solved in the approximation of the kinetic theory (see for instance Chapman \& Cowling 1970), but the complexity of the solution grows rapidly if one increases the number of species in the mixture. In astrophysical situations, one often assumes that the plasma is mainly composed of $\mathrm{H}, \mathrm{He}$, electrons, photons and trace elements (partially ionised). Collisions between charged particles are treated separately from those with photons; sometimes only binary collisions between protons and "other" are explicitely considered. Throughout this Paper, we use the approximate theory developed in Sect. 6.6 of Chapman \& Cowling (1970). This is useful to discuss intuitively the processes. This is not the theory currently used in stellar evolution calculations (e.g. Turcotte et al. 1998; Richer et al. 1998) where the formalism developed by 
Burgers (1969) is used. In other astrophysical applications, the formalism developed in Chapters VIII and following of Chapman \& Cowling (1970) is currently used.

\subsection{Viscosity}

A macroscopic quantity such as viscosity can be interpreted in the framework of the kinetic theory as related to the microscopic momentum transport on a scale length corresponding to a mean free path. Larger is the mean free path, larger is the viscosity, since larger distances in the fluid are connected. This is why the radiative viscosity invoked by Morel $\&$ Thévenin (2002) can be so large when the opacity decreases in the outer layers of a stellar envelope. Therefore, viscosity allows to describe how momentum is homogenized in a fluid. According to the simple reasonning presented by Chapman \& Cowling (1970) in their Sect. 6.63, one can see that contributions of the various gaseous species to the total viscosity are additive. Intuitively, one could say that if one increases the efficiency of momentum transport due to the sth component of the gas, one increases the total momentum transport efficiency (the total viscosity) in proportion to the concentration of that sth component.

\subsection{Atomic diffusion coefficients}

The picture is qualitatively different for the mutual diffusion of species. Following Chapman \& Cowling (1970) (see their Eq. $(6.63,4)$ ), the diffusion velocities $V_{s t}$ of particles $s$ (of mass $m_{s}$ ) with respect to particles $t$ satisfy the following equation:

$\sum_{t} \frac{p_{s} p_{t}}{p D_{s t}} V_{s t}=\mathcal{A}_{s}$

where $p_{t}$ and $p_{s}$ are the partial pressures due to particles $t$ and $s, p$ is the total pressure, $D_{s t}$ the coefficient of mutual diffusion of particles $s$ with respect to particles $t$. The right-hand side term, $\mathcal{A}_{s}$, is a combination of the various forces (such as gravity, electric field, temperature gradient ...) acting on $s$ particles and of the effect of the concentration gradient. Here, for simplicity, we do not detail the various forces (see Sect. 4 where they are included in the diffusion velocity equation).

In order to evaluate how to combine atomic diffusion coefficients, we consider the simple example of a very small concentration of trace ions ( $\mathrm{z}$ ) diffusing in protons (p) and electrons (e). Since we consider trace ions, the diffusion velocity of protons and electrons with respect to the mass is zero, which also implies $V_{\mathrm{pe}}=0$. This allows to consider one single diffusion velocity $V_{\mathrm{z}}$, since $V_{\mathrm{z}}=V_{\mathrm{ze}}=V_{\mathrm{zp}}$. Noting that in our mixture, $p=2 n_{\mathrm{p}} k T$, Eq. (1) leads to:

$V_{\mathrm{z}} \frac{n_{\mathrm{z}} k T}{2}\left(\frac{1}{D_{\mathrm{ze}}}+\frac{1}{D_{\mathrm{zp}}}\right)=\mathcal{A}_{\mathrm{z}}$.

Finally, since $D_{s t} \propto 1 / m_{s} m_{t}$ (see for instance Eq. (6.62,3) of Chapman \& Cowling 1970), $D_{\text {ze }} \gg D_{\text {zp }}$ and we have:

$V_{\mathrm{z}}=\frac{2}{n_{\mathrm{z}} k T} D_{\mathrm{zp}} \mathcal{A}_{\mathrm{z}}$
By this simple example, we have illustrated three facts. The first (i) is that in a multiple mixture of gases (more than two components), the total diffusion coefficient of a given species is obtained through the sum of inverses of coefficients of mutual diffusion, contrary to the case of viscosities. The second (ii) is that, as a consequence of (i), the larger is a coefficient of mutual diffusion, the smaller is its contribution to the total diffusion coefficient. Since coefficients of mutal diffusion are proportional to collision times, the diffusion velocity of $\mathrm{z}$ is dominated by the one of the interactions having the shortest characteristic time (or shorter mean free path), contrary to viscosity. The third fact (iii) is that, regardless of the contribution of a component of a mixture, the total coefficient of diffusion multiplies the whole right-hand side term $\mathcal{A}_{\mathrm{z}}$ which includes all the forces ${ }^{1}$.

These three facts show that, due to (i), if one considers photons as a component of the multiple gas mixture, their effect cannot be reproduced by simply adding a new term to the coefficient of diffusion as suggested by Morel \& Thévenin (2002). Moreover, due to (iii), their effect cannot act only on the concentration gradient term, but must act in the same way on all the forces and on the concentration gradient. Finally as a consequence of (ii), considering that the photons' mean free path increases with increasing distance to the star center, their effect on atomic diffusion velocities cannot have the trend assumed by Morel \& Thévenin (2002).

\section{Photon-ion momentum exchange}

In this section, we evaluate roughly the importance of the photon-ion momentum exchange on atomic diffusion coefficients. We do not claim to make a precise evaluation, which would require the use of a theoretical framework such as developed in Fontenla (1985).

Consider ions $A_{i}$ (with mass $A_{i} m_{\mathrm{p}}$ ) inside a photon gas. We want to evaluate the diffusion coefficient $D_{i, \text { phot }}\left(A_{i}\right)$ of ions $A_{i}$ with respect to photons. One may then use

$D_{i, \text { phot }}\left(A_{i}\right)=\frac{\tau_{i, \text { phot }} k T}{A_{i} m_{\mathrm{p}}}$,

which is equivalent to Eq. $(6.62,3)$ of Chapman \& Cowling (1970) (where we took $\rho=n m_{1}$ and $m_{2}=A_{i} m_{\mathrm{p}}$ ).

The approach we follow implicitly assumes that the relaxation and slowing down times are of the same order, as Spitzer (1968), for instance, shows to be approximately the case. We assume that $\tau_{i \text {,phot }}$ is the slowing down time needed for $A_{i}$ to loose the momentum $A_{i} m_{\mathrm{p}} v$ due to momentum exchange with the isotropic part of the radiation field. We define $g_{\text {rad,slow }}$ as the average deceleration which particles $A_{i}$ undergo through interactions with photons, and then one has:

$\tau_{i, \text { phot }}=\frac{v}{g_{\text {rad,slow }}}$.

In evaluating the effect of photon-ion momentum exchange, it is useful to consider separately the interaction with the

\footnotetext{
${ }^{1}$ Here we discuss atomic diffusion, the turbulent diffusion coefficient will be discussed in Sect. 4.
} 
anisotropic and isotropic parts of the photon distribution. The interaction between ion $A_{i}$ and the anisotropic part of the photon distribution leads to radiative accelerations of $A_{i}, g_{\mathrm{rad}}\left(A_{i}\right)$ (Michaud 1970), which one evaluates using:

$g_{\mathrm{rad}}\left(A_{i}\right)=\frac{1}{A_{i} m_{\mathrm{p}} c} \int_{0}^{\infty} \sigma_{v}\left(A_{i}\right) F_{\nu} \mathrm{d} v$,

where $\sigma_{v}$ is the absorption cross section and $F_{v}$ the net photon flux at frequency $v$.

However except in the outer stellar atmosphere, the photon distribution is mainly isotropic so that most ion-photon interactions are with the isotropic part of the photon distribution which may be approximated by the Planck function, $B_{v}$, at the local temperature $T$. One may then consider the photons to have a spherically symmetrical distribution in the stellar rest frame and evaluate how they slow down an ion that has a velocity $v$ with respect to the rest frame ${ }^{2}$.

We first describe the radiation field seen by a moving particle inside a black body. We approximate the angular integration of the interaction in the ion frame by the difference of the cross-section integrated over the Plank function in respectively the forward and backward directions. The situation is then analogous to that of a moving observer with velocity $v$, inside the cosmic microwave background as studied by Peebles \& Wilkinson (1968) who were interested in the Planck function of the primeval fireball. The well known result is that the black body appears hotter (by $\mathrm{d} T / T=+v / c$, to first order in $v / c$ ) in the direction of the movement and cooler in the opposite one. As stressed by Peebles \& Wilkinson (1968), this is "at any wavelength" in the observer reference frame. For the forward looking part of the integration of the cross-section, we use the "hotter" blackbody $\left(B_{v \mathrm{~h}}\right)$ and, for the backward looking part, we use the "cooler" one $\left(B_{v \mathrm{c}}\right)$.

To further simplify the argument, we assume that photons are streaming either parallel or antiparallel to the ion. It is then the difference in the momentum exchanged between photon stream with energy distribution given by the "hotter" blackbody $\left(B_{v \mathrm{~h}}\right)$ minus the momentum exchanged with the "cooler" one $\left(B_{v_{\mathrm{c}}}\right)$ that slows down the ions. In this simplified situation, we can use Eq. (6) and write:

$g_{\text {rad,slow }} \approx \frac{1}{A_{i} m_{\mathrm{p}} c} \int_{0}^{\infty} \frac{1}{2} \sigma_{v}\left(B_{v \mathrm{~h}}-B_{v \mathrm{c}}\right) \mathrm{d} v$,

where, comparing to Eq. (6), $\left(B_{v \mathrm{~h}}-B_{v \mathrm{c}}\right)$ may be interpreted as an apparent flux induced by the velocity, $v$, and where $B_{v \mathrm{~h}}$, according to Peebles \& Wilkinson (1968), is given by:

$B_{v \mathrm{~h}}=B_{v}(T(1+v / c)) \approx B_{v}(T)+\frac{v}{c} T \frac{\partial B_{v}}{\partial T}$.

\footnotetext{
2 This change of reference frame is also the origin of the momentum transfer in the relaxation time evaluations due to photons made by Einstein (1917, Sect. 5) and Oxenius (1986) Sect. 7.2.
}

A similar expression is obtained for $B_{v c}$ with $-v$. From Eqs. (4), (5), (7) and (8) we have finally:

$D_{i, \mathrm{phot}} \approx k c^{2}\left[\int_{0}^{\infty} \sigma_{v} \frac{\partial B_{v}}{\partial T} \mathrm{~d} v\right]^{-1}$.

As with all mutual diffusion coefficients, this quantity does not depend on the velocity $v$. One can evaluate directly the integral for a resonance line having an oscillator strenght $f$ at the frequency where $\frac{\partial B_{v}}{\partial T}$ has its maximum.

For one line, at frequency $v_{0}$ and assuming $\frac{\partial B_{v}}{\partial T}$ constant over the width of the line, one may use $\int \sigma \mathrm{d} v=\left(\pi e^{2} / m_{\mathrm{e}} c\right) f$ to obtain:

$\int_{0}^{\infty} \sigma_{v} \frac{\partial B_{v}}{\partial T} \mathrm{~d} v \approx\left(\pi e^{2} / m_{\mathrm{e}} c\right) f \frac{\partial B_{v_{0}}}{\partial T}$

Using $\frac{\partial B_{v_{0}}}{\partial T}$ evaluated at $h v_{0} / k T \sim 4$, which is close to the value for the frequency where $\frac{\partial B_{v_{0}}}{\partial T}$ has its maximum, one obtains:

$\int_{0}^{\infty} \sigma_{v} \frac{\partial B_{v}}{\partial T} \mathrm{~d} v \approx\left(\pi e^{2} / m_{\mathrm{e}} c\right) f\left[10 k\left[\frac{k T}{h c}\right]^{2}\right]$.

Equation (9) then leads to

$D_{i, \text { phot }}\left(A_{i}\right) \approx \frac{10^{23}}{f T^{2}}$,

in the cgs system.

A different way to estimate this quantity in stellar interiors is given in the Appendix. At temperature $T=10^{5} \mathrm{~K}$, one obtains $D_{i \text {,phot }}\left(A_{i}\right) \approx 10^{12}$ in the cgs system even if $f=10$. This is some 10 orders of magnitude larger than the atomic diffusion coefficient of $\mathrm{Fe}$ in a stellar plasma at the same temperature. Using $f=10$ nearly certainly overestimates the integral since the sum of all lines in the appropriate part of the spectrum rarely exceeds 1.0 in practice (see the discussion in Appendix A of Michaud et al. 1976).

Given Eq. (2), $D_{i, \text { phot }}\left(A_{i}\right)$ may be safely neglected. Consequently, the effects on the particle transport due to the interaction between $A_{i}$ and the isotropic part of the photon gas are negligible. The interaction with the anisotropic part of the photon gas is taken into account by using $g_{\text {rad }}$ (see Eq. (6)) in the diffusion velocity equation (see the next section).

\section{The turbulent "diffusion" coefficient}

In order to understand Morel \& Thévenin (2002) work, it is convenient to use a diffusion equation developed for ternary mixtures by Aller \& Chapman (1960) to which one must add differential radiative accelerations, $g_{\mathrm{rad}}$, (Michaud 1970) as well as a the effect of turbulence.

Schatzman (1969) evaluated how macroscopic turbulent motions reduce abundance anomalies caused by gravitational settling. The random turbulent motions tend to homogenize any abundance variations caused by gravitational settling. Tubulence is the velocity field of a large spectrum of mostly 
advective motions. Using the continuity equation, Schatzman showed that the effect of those random turbulent motions could be modeled by adding a turbulent diffusion coefficient to the atomic diffusion coefficient in front of the purely diffusive term (see his Eqs. (17) and (18)) in the Aller \& Chapman (1960) diffusion velocity equation. His results may be expressed as:

$$
\begin{aligned}
v_{D}=( & \left.D_{i p p}+D_{\mathrm{T}}\right)\left[-\frac{\partial \ln c_{i}}{\partial r}\right] \\
& +D_{i p p}\left[\frac{A_{i} m_{\mathrm{p}}}{k T}\left(g_{\mathrm{rad}, i}-g\right)+\frac{Z_{i} m_{\mathrm{p}} g}{2 k T}+k_{\mathrm{T}} \frac{\partial \ln T}{\partial r}\right] .
\end{aligned}
$$

Element $A_{i}$ is assumed to be a trace element of small abundance, $c_{i}$, with respect to protons. Within the first brackets of the right hand side of the equation is the purely diffusive term which includes a contribution both from atomic diffusion, $D_{i p p}$, and from turbulent macroscopic mixing, $D_{\mathrm{T}}$. Note that $D_{\mathrm{T}}$ is approximately the same for all particles ${ }^{3}$. Within the second brackets of the right hand side, is an advective term caused by radiative acceleration, gravity, electric field, and thermal diffu$\operatorname{sion}^{4}$.

In evolutionary model calculations, the second term in the right side of Eq. (13), the advective one, leads to the appearance of abundance anomalies, i.e. it builds abundance gradients within the star. The first term on the right limits them and usually has the opposite sign to the advective term.

The photon distribution is nearly isotropic in the star's reference frame. If one adds the interaction with the photon gas, the process is similar as done in Sect. 2 for electrons. Since $D_{i, \text { phot }}\left(A_{i}\right)$ is very large (see Sect. 3), according to Eq. (2) it contributes little to the total atomic diffusion coefficient, $D_{i p p}$, that appears in Eq. (13). This small contribution of the interaction with photons in $D_{i p p}$ affects all the terms on the right. As such, adding the interaction with the isotropic radiation field does not significantly modify the equilibrium reached. It only slightly modifies the diffusion velocity and so the time it takes to reach equilibrium. Because the coupling to the photon gas is weak, as shown in Sect. 3, adding it hardly modifies the diffusion velocity.

One may now interpret the results of the stellar evolution calculations of Morel \& Thévenin (2002). Since the term they add (the radiative diffusivity) reduces the abundance anomalies reached in their calculations, they presumably include it as a turbulent diffusion, $D_{\mathrm{T}}$, in Eq. (13). They probably add to the particle transport equation a turbulent term which they assume proportional to radiative viscosity.

\section{Conclusion}

It has been shown that the so called radiative diffusivity introduced by Morel \& Thévenin (2002) is not introduced as a correction to atomic diffusion coefficients. The analogy the authors use to justify the process is erroneous. However given the

\footnotetext{
3 The situation is more complex if one considers anisotropic turbulence. See for instance Vincent et al. (1996).

4 We have kept only the dominant terms in the second bracket. The term involving $Z_{i}$ comes from the electric field in an electron proton plasma assuming only gravity separates protons from electrons
}

current uncertainty on the origin of turbulence in stars, it is not inappropriate to investigate alternate parametrizations to those generally considered. As such the evolutionary models calculated by the authors may be viewed as testing the effect of a turbulent transport coefficient, $D_{\mathrm{T}}$, assumed proportional to radiative viscosity.

Finally we propose an intuitive explanation of why radiative viscosity dominates molecular viscosity while interaction with the isotropic part of the photon fluid has a negligible effect on atomic diffusion. The effect of viscosity is to transport momentum between layers. This depends on the mean free path of the transporting agent. Photons have a much larger mean free path than protons and so can link relatively far away layers. Atomic diffusion coefficients, on the other hand, depend only on local collisions. The small momentum exchange efficiency of collisions with photons is compensated for viscosity by the greater photon mean free path (compared to protons) so that interacting photons carry momentum from relatively far away layers having a relatively large velocity difference with the local layer; however this plays no role for the atomic diffusion coefficient. Similarly the relatively long electron mean free path explains why electrons play a subtantial role for the thermal diffusion coefficient even if they play a negligible one for the atomic diffusion coefficient (see Sect. 4 of Michaud 1991).

Acknowledgements. We thank the referee, Dr Fontenla, for numerous suggestions and for voicing objections that led to significant improvements of this paper. This work has been supported by an Operating Grant to G.M. from the Natural Sciences and Engineering Research Council of Canada, and the Visiting Astronomer programme at the Observatoire de Paris-Meudon. We thank Paul Charbonneau and Gérard Massacrier for useful discussions.

\section{Appendix A: Alternate numerical estimate of $D_{i, \text { phot }}$}

One notices that the integral in Eq. (9) is almost the same as the one which must be computed for $g_{\mathrm{rad}}\left(A_{i}\right)$ if the opacity due to $A_{i}$ does not dominate the total opacity at any frequency (the unsaturated case). One may then lean on the large number of available calculations of $g_{\mathrm{rad}}\left(A_{i}\right)$ for elements with small abundance to evaluate the integral. In stellar interiors, the net flux (in the well known diffusion approximation) is given by:

$F_{v}=-\frac{4 \pi}{3} \frac{1}{\rho \kappa_{v}} \frac{\partial B_{v}}{\partial T} \frac{\partial T}{\partial r}$

Assuming that the radiation flux is conserved throughout the star, one can relate this flux to the emergent flux, and then to the effective temperature ( $\left.T_{\text {eff }}\right)$. Using the definition of $T_{\text {eff }}$, Eq. (A.1) becomes:

$F_{v} \approx \frac{\pi}{4} \frac{\kappa_{R}}{\kappa_{v}} \frac{T_{\mathrm{eff}}^{4}}{T^{3}}\left(\frac{R}{r}\right)^{2} \frac{\partial B_{v}}{\partial T}$,

where $\kappa_{R}$ and $\kappa_{v}$ are respectively the local Rosseland and monochromatic opacities, $R$ and $r$ are respectively the total and local stellar radius. 
According to Eq. (6), assuming $R / r \approx 1$, that the absorption lines are unsaturated and that $\kappa_{R} / \kappa_{v} \approx 1$, one can express the radiative acceleration as:

$g_{\text {rad,unsaturated }} \approx \frac{\pi}{4} \frac{1}{A_{i} m_{\mathrm{p}} c} \frac{T_{\mathrm{eff}}^{4}}{T^{3}} \int_{0}^{\infty} \sigma_{v} \frac{\partial B_{v}}{\partial T} \mathrm{~d} v$.

The radiative accelerations for unabundant atomic species with properly placed absorbing lines have been evaluated by Michaud et al. (1976) and their Eq. (6) gives in cgs units at $r=R$ :

$g_{\text {rad,unsaturated }} \approx 1.7 \times 10^{-4} \frac{T_{\text {eff }}^{4}}{A_{i} T}$.

Equating the last two equations, leads to an evaluation of the integral appearing in Eq. (A.3). Using this value for the integral appearing in Eq. (9), one obtains:

$D_{i, \mathrm{phot}}\left(A_{i}\right) \approx \frac{10^{22}}{T^{2}}$

in the cgs system which is the same as Eq. (12) with $f=10$.

Since the paper Michaud et al. (1976) appeared, many more accurate radiative accelerations have been calculated, as described in Richer et al. (1998), in stellar evolution programs. In practice they all turned out to be smaller or of the order of the estimate of Eq. (6) of Michaud et al. (1976). This confirms the expectation that Eq. (A.5), or equivalently Eq. (12), should give a lower limit to $D_{i, \text { phot }}\left(A_{i}\right)$.

\section{References}

Aller, L. H., \& Chapman, S. 1960, ApJ, 132, 461

Burgers, J. M. 1969, Flow Equations for Composite Gases (New York: Academic Press)

Chapman, S., \& Cowling, T. G. 1970, The Mathematical Theory of non-uniform Gases, 3rd ed. (Cambridge: Cambridge University Press)

Einstein, A. 1917, Phys. Z., XVIII, 121

Fontenla, J. M. 1985, Rev. Mex. Astron. Astrofis., 10, 413

Michaud, G. 1970, ApJ, 160, 641

Michaud, G. 1991, Ann. Phys. Paris, 16, 481

Michaud, G., Charland, Y., Vauclair, S., \& Vauclair, G. 1976, ApJ, 210, 447

Morel, P., \& Thévenin, F. 2002, A\&A, 390, 611

Oxenius, J. 1986, Kinetic theory of particles and photons. Theoretical foundations of Non-LTE plasma spectroscopy Springer Series in Electrophysics (Berlin: Springer)

Paquette, C., Pelletier, C., Fontaine, G., \& Michaud, G. 1986, ApJS, 61, 177

Peebles, P. J., \& Wilkinson, D. T. 1968, Phys. Rev., 174, 2168

Richer, J., Michaud, G., Rogers, F., et al. 1998, ApJ, 492, 833

Schatzman, E. 1969, A\&A, 3, 331

Spitzer, L. 1968, Diffuse Matter in Space (New York: Interscience Publishers)

Turcotte, S., Richer, J., Michaud, G., Iglesias, C., \& Rogers, F. 1998, ApJ, 504, 539

Vincent, A., Michaud, G., \& Meneguzzi, M. 1996, Phys. Fluids, 8, 1312 